\title{
Bifurcation and Chaos in Integer and Fractional Order Two-Degree-of-Freedom Shape Memory Alloy Oscillators
}

\author{
Karthikeyan Rajagopal (D), ${ }^{1,2}$ Anitha Karthikeyan, ${ }^{1,2}$ Prakash Duraisamy, \\ and Riessom Weldegiorgis ${ }^{2}$ \\ ${ }^{1}$ Center for Nonlinear Dynamics, Department of Electrical and Communication Engineering, The PNG University of Technology, \\ Lae, Papua New Guinea \\ ${ }^{2}$ Center for Nonlinear Dynamics, Defence University, Bishoftu, Ethiopia
}

Correspondence should be addressed to Karthikeyan Rajagopal; rkarthiekeyan@gmail.com

Received 2 November 2017; Accepted 4 January 2018; Published 31 January 2018

Academic Editor: Viorel-Puiu Paun

Copyright (c) 2018 Karthikeyan Rajagopal et al. This is an open access article distributed under the Creative Commons Attribution License, which permits unrestricted use, distribution, and reproduction in any medium, provided the original work is properly cited.

A two-degree-of-freedom shape memory oscillator derived using polynomial constitutive model is investigated. Periodic, quasiperiodic, chaotic, and hyperchaotic oscillations are shown by the shape memory alloy based oscillator for selected values of the operating temperatures and excitation parameters. Bifurcation plots are derived to investigate the system behavior with change in parameters. A fractional order model of the shape memory oscillator is presented and dynamical behavior of the system with fractional orders and parameters are investigated.

\section{Introduction}

A shape memory alloy (SMA) shows temperature and stress induced martensitic phase transformation, drastically changing the material's mechanical properties. In other words, after an apparent plastic deformation, it will return to their original shape when heated. The same materials, in a certain temperature range, can be strained up to approx. $10 \%$ and still will return to their original shape when unloaded. These unusual effects are called thermal shape memory and superelasticity (elastic shape memory), respectively, which can use both sensor and actuator [1]; it is increasing range of potential applications, such as Biomedical [2], Robotics [3], and Aerospace. Stress-strain curve study reveals hysteretic phenomenon of SMAs. Hysteretic effect leads to nonlinearity. This property results in high damping capacity [2] which can be utilized for amplitude attenuation in vibrating system [4] so the application of these materials can be extended to control elements too.

Various constitutive models of shape memory oscillators (SMO) have been developed since 1990, in order to analyze the dynamical behavior [5-9]. The study reveals that SMOs can expose to chaotic region when it is treated as single degree-of-freedom system but can extend to hyperchaos when it is treated as two-degree-of-freedom system [10,11]. Similar property is noted down in other SMAs. According to Falk model strain and temperature are the only state variables; free energy is validated for various temperatures and shows unstable points and is not considering twinned martensite. Brinson's model describes the reorientation process clearly compared to other models, the numerical simulations reveals the internal subloops present in thermomechanical [12] loading. Tanaka model considered martensitic volumetric fraction so it can describe compressive behavior accurately [13]. The transient response and attractor's multistability are interesting property in nonlinear study, and the numerical analysis of SMOs nonlinear models shows these properties significantly [10]. Dynamical jumps of SMOs were studied and found that the response is not only depending on the forcing amplitude but also on the way the forcing frequency is modified [14].

Experimental dynamic analysis of SMAs was carried out and found nonlinearity in phase transformation leads to complicated dynamic behavior of the system [15]. Nonlinear dynamics of SMAs are investigated experimentally and it is noted that the actuator response shows unpredictability, 


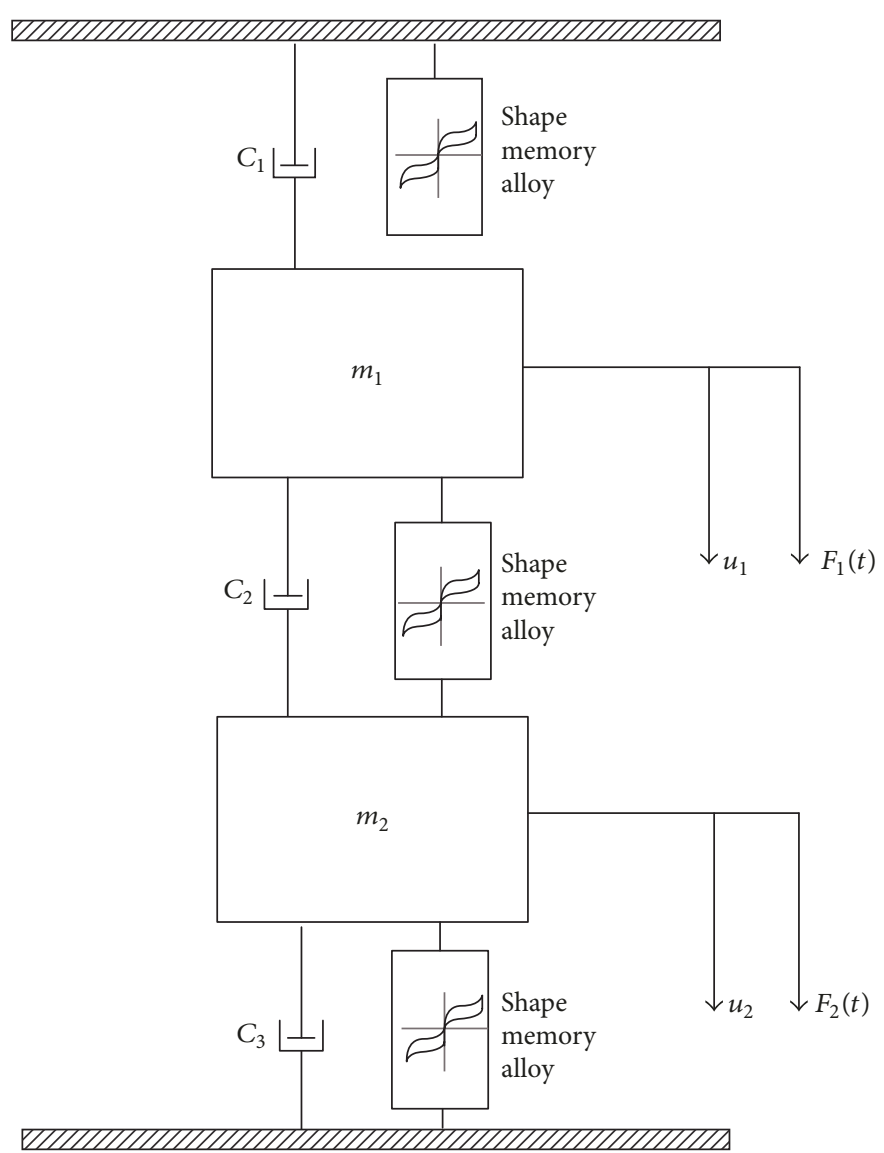

FIGURE 1: The two-degree-of-freedom oscillator with shape memory alloys.

when low-to-moderate voltage was applied to the system and added that could affect the system design and can cause control difficulties in precise applications [3].

The real systems are fractional in nature, so there is no surprise on treatment of fractional order describing behavior of the system more accurate than the integer methods. Because of holding nonlocal property, that is, the next state of a system depends not only upon its current state but also upon all of its historical states, fractional calculus can provide more realistic results. Recently fractional order calculus used to refine the results in various fields like thermodynamics, mechatronics systems, chaos theory, and biomedical system as well. When a chaotic nonlinear system is treated as integer order, it demands minimum order of 3 for chaos to appear [16]. Conversely while the system is treated as fractional order, chaos can be identified with lesser order. For example, Chau's circuit with order 2.7 can produce chaotic attractor [17]. Recently the active and passive vibration damping capacity of SMAs are studied. Oberaigner model has been taken, and fractional order results reveal the chaotic behaviors precisely [18]. The generalized theory of electrothermoelasticity of fractional order heat transfer describes the behavior of the particles of an elastic body more realistically than the theory of generalized thermoelasticity with integer order [19]. Yu et al. developed the theory of fractional order generalized electro-magneto-thermoelasticity for anisotropic and linearly electro-magneto-thermo-elastic media and extended that the fractional order has great effect on the response when the material is imposed a sudden heating [20].

\section{Dynamics of the Two-Degree-of-Freedom Shape Memory Alloy (2DOF SMA) Oscillator}

Even though the authors of [11] have done a [0-1] test on different models of SMAs, the complete dynamical analysis of the system is less investigated. Hence we are interested in analyzing the two-degree-of-freedom (2DOF) SMA [21] shown in Figure 1 because of its higher dimension and complex behaviors.

The dimensionless mathematical model of the 2DOF SMA polynomial constitutive model can be derived using the equation of motions and is given by $[11,21]$

$$
\begin{aligned}
\dot{x} & =y, \\
\dot{y} & =\left[\delta_{1} \sin \left(\omega_{1} t\right)-\left(\varepsilon_{1}+\varepsilon_{2} v_{21} \mu\right) y+\varepsilon_{2} v_{21} \mu z\right. \\
& -\left[\left(\theta_{1}-1\right)+v_{21}{ }^{2} \mu\left(\theta_{2}-1\right)\right] x+v_{21}{ }^{2} \mu\left(\theta_{2}-1\right) z \\
& +\phi_{1} x^{3}-\lambda_{1} x^{5}-\phi_{2} v_{21}{ }^{2} \mu(z-x)^{3} \\
& \left.+\lambda_{2} v_{21}{ }^{2} \mu(z-x)^{5}\right], \\
\dot{z} & =w,
\end{aligned}
$$


TABLE 1: Types of SMA behavior.

\begin{tabular}{lcc}
\hline Parameters & Type of SMA behavior & Lyapunov exponents \\
\hline$\theta_{1}=0.7, \theta_{2}=3.5, \theta_{3}=0.7$ & Periodic & $-0.128 ;-0.140 ;-0.257 ;-0.275$ \\
$\theta_{1}=1.5, \theta_{2}=3.5, \theta_{3}=1.5$ & Quasiperiodic & $0 ;-0.209 ;-0.265 ;-1.272$ \\
$\theta_{1}=1.5, \theta_{2}=0.5, \theta_{3}=1.5$ & Chaotic & $0.378 ; 0 ;-0.438 ;-0.786$ \\
$\theta_{1}=1.5, \theta_{2}=1.5, \theta_{3}=1.5$ & Hyperchaotic & $0.453 ; 0.233 ;-0.651 ;-0.835$ \\
\hline
\end{tabular}

$$
\begin{aligned}
\dot{w} & =\left[\alpha_{21}{ }^{2} \delta_{2} \sin \left(\omega_{2} t\right)+\varepsilon_{2} v_{21} y-\left(\varepsilon_{2} \alpha_{21}+\varepsilon_{3} v_{21} \alpha_{32}\right) w\right. \\
& +v_{21}{ }^{2}\left(\theta_{2}-1\right) x \\
& -\left[v_{21}{ }^{2}\left(\theta_{2}-1\right)+v_{21}{ }^{2} v_{32}{ }^{2}\left(\theta_{3}-1\right)\right] z \\
& +\phi_{2} v_{21}{ }^{2}(z-x)^{3}-\lambda_{2} v_{21}{ }^{2}(z-x)^{5}+\phi_{3} v_{21}{ }^{2} v_{32}{ }^{2} z^{3} \\
& \left.-\lambda_{3} v_{21}{ }^{2} v_{32}{ }^{2} z^{5}\right],
\end{aligned}
$$

where $\delta_{1}, \delta_{2}, \omega_{1}, \omega_{2}$ are the excitation parameters, $\varepsilon_{1}, \varepsilon_{2}, \varepsilon_{3}$ are the dissipation parameters, $\mu$ is the mass relation parameter, and $v_{21}, v_{32}, \alpha_{21}, \alpha_{32}, \phi_{1}$, $\phi_{2}, \phi_{3}, \lambda_{1}, \lambda_{2}, \lambda_{3}$ are the SMA properties. The states $x$ and $z$ are the dimensionless displacements, and $y$ and $w$ are the dimensionless velocity components of the 2DOF SMA. For the parameter values,

$$
\begin{aligned}
\omega_{1} & =\omega_{2}=1, \\
\varepsilon_{1} & =\varepsilon_{2}=\varepsilon_{3}=0.2, \\
\phi_{1} & =\phi_{2}=\phi_{3}=1.3 e+3, \\
\lambda_{1} & =\lambda_{2}=\lambda_{3}=4.7 e+5, \\
v_{21} & =v_{32}=1, \\
\mu & =1, \\
\delta_{1} & =0.06, \\
\delta_{2} & =0, \\
\alpha_{21} & =\alpha_{32}=1,
\end{aligned}
$$

and depending on the temperatures of the SMA given by the parameters $\theta_{1}, \theta_{2}, \theta_{3}$, the system changes the character as periodic, quasiperiodic, chaotic, and hyperchaotic [11]. Table 1 shows the different types of behavior of the SMA with various values of $\theta_{1}, \theta_{2}, \theta_{3}$ with the respective finite time Lyapunov exponents (LEs) calculated using Wolf algorithm [22] for 20000s.

Figure 2 shows the $2 \mathrm{D}$ phase portraits of the SMA system with initial conditions $[0,0,0,1]$ for various values of $\theta_{1}, \theta_{2}, \theta_{3}$ as given in Table 1 . It can be observed from Figure 2 that the SMA system shows periodic, quasiperiodic, chaotic, and hyperchaotic behaviors.

Bifurcation. Bifurcation analysis is helpful in investigating the qualitative changes in the states of a system with respect to the variation in a parameter [23]. Recent techniques have shown that bifurcation analysis can be done with experiments rather than only mathematical methods. Both continuous and discrete models can be analyzed by bifurcations. To analyze the 2DOF SMA using bifurcation plots, we fix all the other parameters like in (2) and consider $\theta_{2}$ as the bifurcation parameter. The initial conditions for the first iteration are taken as $[0,0,0,1]$ and are reinitialized to the end values of state trajectories at each iteration. Figure 3(a) shows the bifurcation diagram of system (1) with respect to the parameter $\theta_{2}$. The parameter values of the SMA system are taken as in (2) with $\theta_{1}=1.5, \theta_{3}=1.5$, and initial conditions for the first iteration are taken as $[0,0,0,1]$ and are reinitialized to the end values of the state variables at the end of each iteration. It can be clearly observed from Figure 2(a) that the SMA shows periodic, quasiperiodic, chaotic, and hyperchaotic oscillations. For $\theta_{2}<0.45$ the SMA system shows periodic limit cycles. For $2.9 \leq \theta_{2} \leq 3.75$ the SMA systems show quasiperiodic/torus attractor. Figure 3(b) shows the bifurcation of 2DOF SMA for parameter $\theta_{1}$ with $\theta_{2}=1.5, \theta_{3}=1.5$ which shows periodicity, chaos, hyperchaos, and quasiperiodicity. Period 2 limit cycles are seen for $0 \leq \theta_{1} \leq 0.75$, period 3 limit cycles are exhibited for $2.2<\theta_{1} \leq 2.5$ and $3<\theta_{1} \leq 3.25$, and period 4 limit cycles are seen for $4.3 \leq \theta_{1} \leq 4.5$. Chaotic oscillations are seen when $0.75<\theta_{1} \leq 1.25,1.8<\theta_{1} \leq 2.2,3.25<\theta_{1} \leq 3.5$, and $3.75<\theta_{1} \leq 4.3$ and hyperchaos is seen for a small range of $1.25<\theta_{1} \leq 1.8$. Quasiperiodic oscillations are shown for $3.5<\theta_{1} \leq 3.75$. Figure 3 (c) shows the bifurcation of the 2DOFSMA system with $\delta_{1}$ and $\theta_{1}=1.5, \theta_{2}=1.5, \theta_{3}=$ 1.5. Chaotic and hyperchaotic regions are seen in the region $0.048<\delta_{1} \leq 0.075$ and chaotic region for $0.035<\delta_{1} \leq 0.045$. For $\delta_{1}<0.018$ we could observe period 1 limit cycles and quasiperiodic oscillations for $0.018<\delta_{1} \leq 0.03$.

\section{Fractional Order Two-Degree-of- Freedom Shape Memory Alloy (FO2DOFSMA) Oscillator}

Fractional order calculus is as old as integer order as seen from a letter written by Leibniz to L'Hopital [24]. A more common form of general order is given by

$$
D^{q}= \begin{cases}\frac{d^{q}}{d t^{q}} & q<0 \\ 1 & q=0 \\ \int_{a}^{t} d \tau^{-q} & q>0\end{cases}
$$

where $q$ is the order. The three ways for simulating fractional order systems [25] are computational methods based on the 

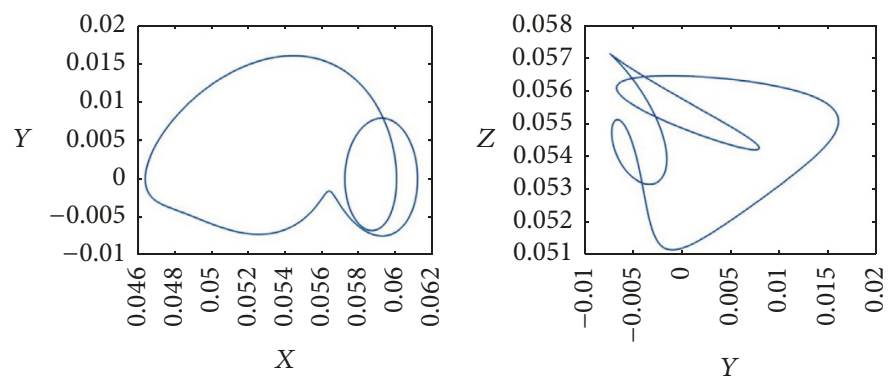

(a)
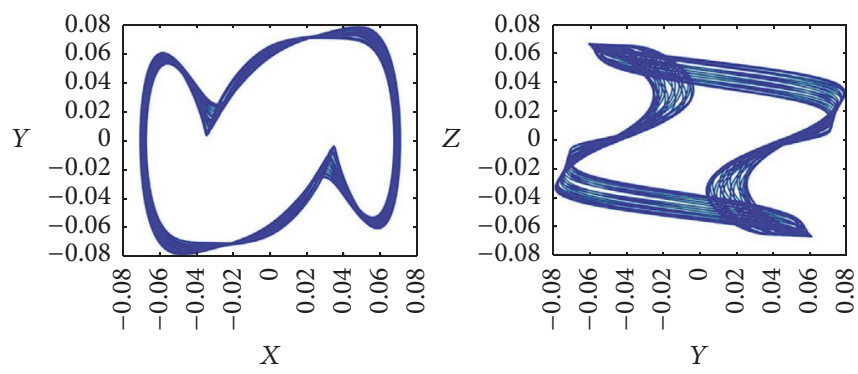

(b)
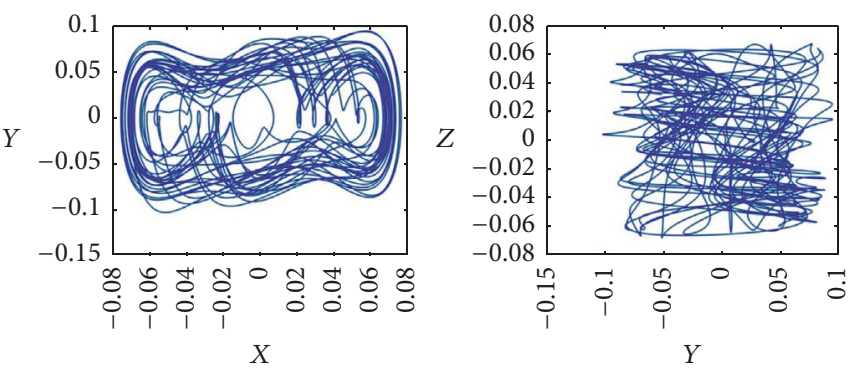

(c)

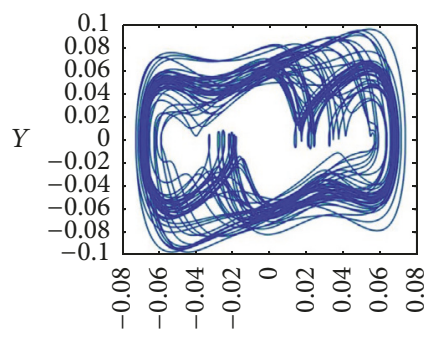

$X$

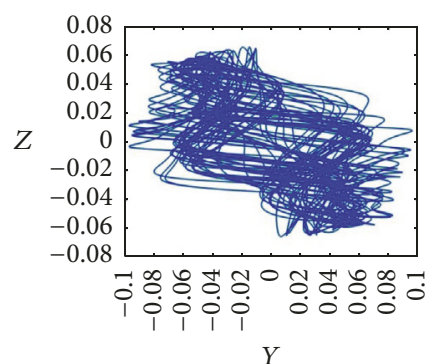

(d)
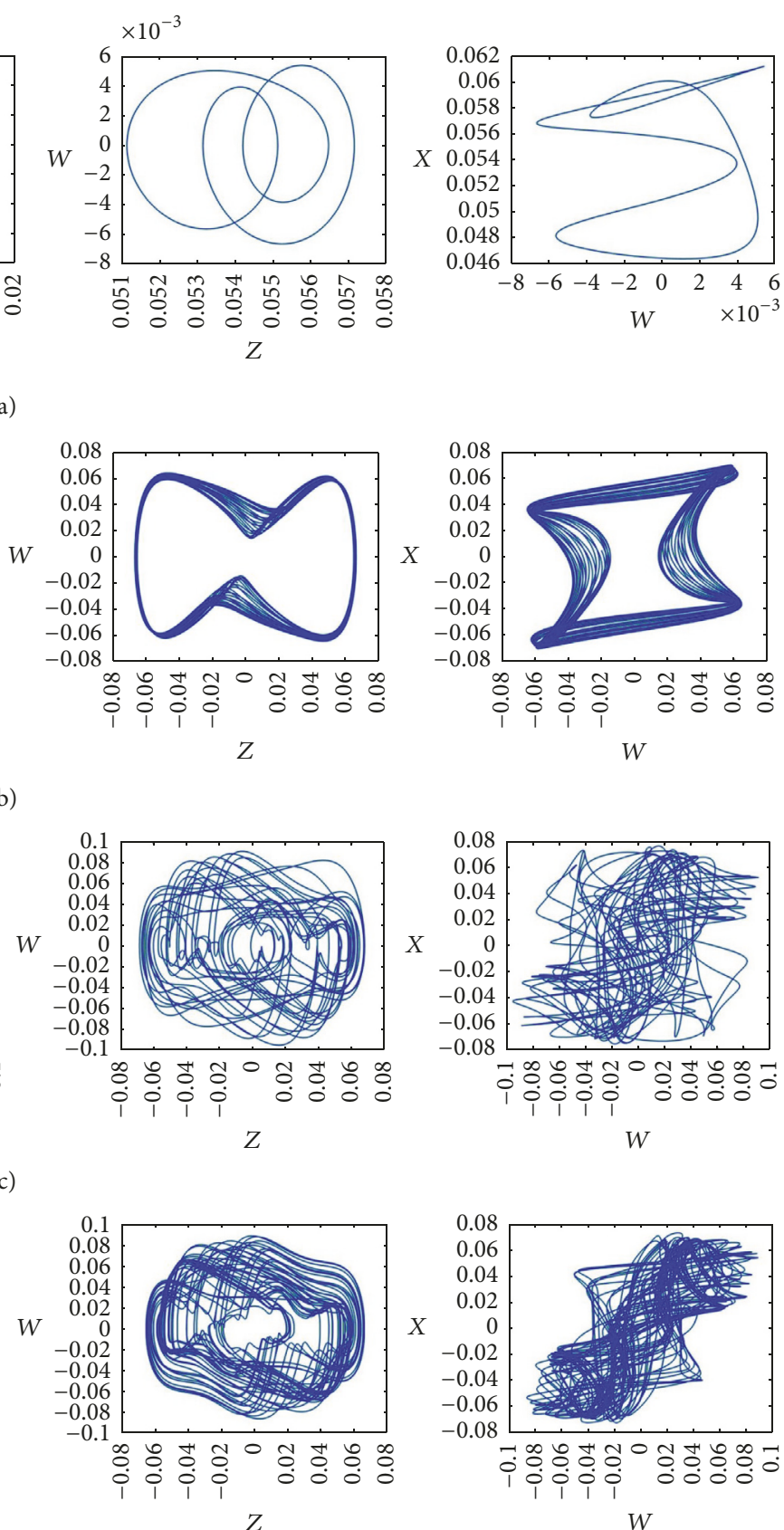

W

FIGURE 2: 2D phase portraits of the 2DOF SMA system: (a) periodic; (b) quasiperiodic; (c) chaotic; (d) hyperchaotic.

mathematical equations, approximation through a rational system in discrete time, and approximation of the fractional system using rational function in continuous time. Most researchers prefer approximation through a rational system in discrete time in which the fractional order system is replaced by its discrete equivalent and approximated again by truncating the polynomial series, which reduces the requirement of infinite memory.

Many different approaches for numerical simulations of fractional order systems have been extensively investigated [26-28]; however there is always a tradeoff between computational efficiency, complexity, and the accuracy of the approximations. Recent researchers have been working on developing fast convolution quadrature algorithms relevant to fractional differential equations because fractional calculus operators work either in continuous or discrete convolution of some form.

There are three commonly used definitions of the fractional order differential operator: Grünwald-Letnikov, Riemann-Liouville, and Caputo [23, 29, 30]. We used the Grünwald-Letnikov (GL) definition to derive the fractional order model of 2DOFSMA. The Grünwald-Letnikov method 


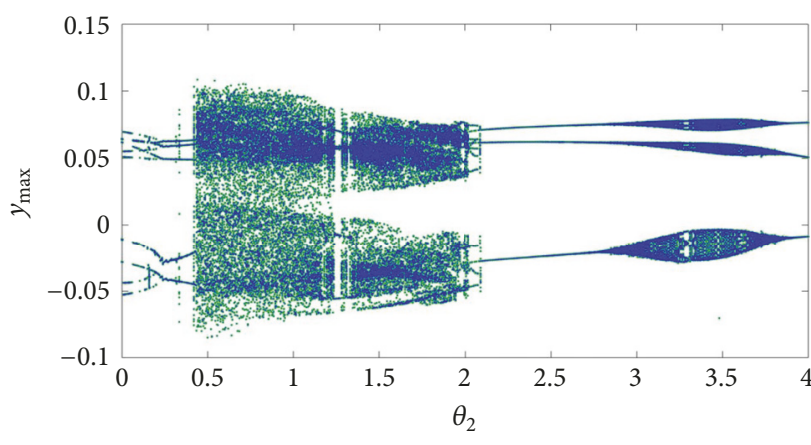

(a)

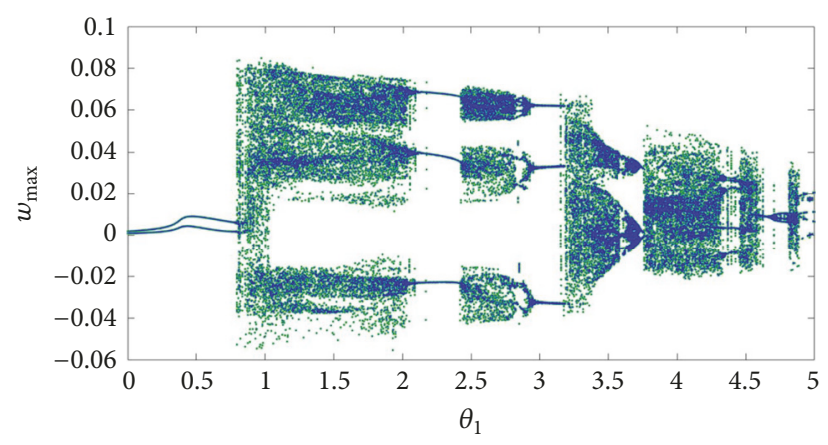

(b)

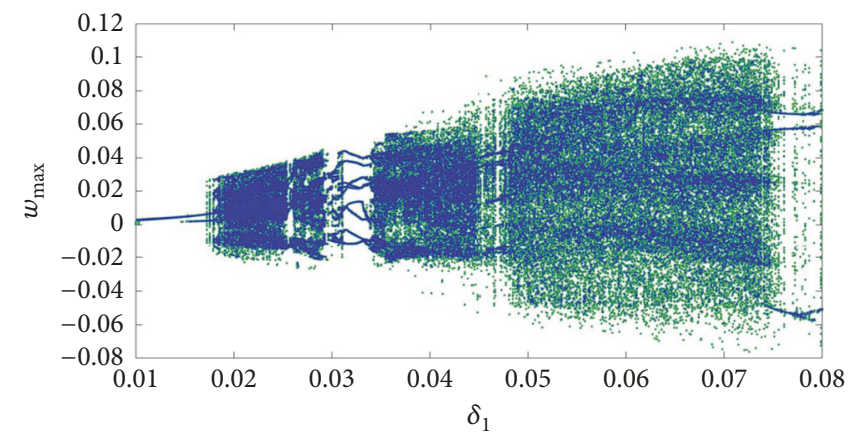

(c)

FIGURE 3: Bifurcation plots of 2DOF SMA with (a) $\theta_{2}$, (b) $\theta_{1}$, and (c) $\delta_{1}$ with initial conditions $[0,0,0,1]$.

is proceeding iteratively but the sum in the scheme becomes longer and longer, which reflects the memory effect. The binomial coefficients are recursively defined and show very smooth properties. The Grünwald-Letnikov derivative is used in many numerical schemes for discretizing fractional diffusion equations from a continuous Riemann-Liouville approach [31-33] and involves a discrete convolution between a "weight" or binomial coefficient function and the function of interest for differentiation. The analytical model of the binomial coefficients is well established in the literature [33].

Most of the literatures have used the Caputo method for numerical solutions of the fractional order systems. But GL method has benefits over the other methods of solving fractional orders due to the smoothness of the resultant approximations [34]. Hence we use the GL method to derive the fractional order 2DOF SMA. The GL derivative can be defined as

$$
\begin{aligned}
{ }_{a} D_{t}^{q} f(t) & =\lim _{h \rightarrow 0}\left\{\frac{1}{h^{q}} \sum_{j=0}^{[(t-a) / h]}(-1)^{j}\left(\begin{array}{l}
q \\
j
\end{array}\right) f(t-j h)\right\} \\
& =\lim _{h \rightarrow 0}\left\{\frac{1}{h^{\alpha}} \Delta_{h}^{q} f(t)\right\},
\end{aligned}
$$

where $a$ and $t$ are limits of the fractional order equation, $\Delta_{h}^{q} f(t)$ is generalized difference, $h$ is the step size, and $q$ is the fractional order of the differential equation.
For numerical calculations the above equation is modified as

$$
{ }_{(t-L)} D_{t}^{q} f(t)=\lim _{h \rightarrow 0}\left\{h^{-q} \sum_{j=0}^{N(t)} \beta_{j}(f(t-j h))\right\} .
$$

We use the short memory principle to limit the required memory for binomial coefficients using

$$
N=\min \left\{\left[\frac{t}{h}\right],\left[\frac{L}{h}\right]\right\}
$$

The binomial coefficients required for the numerical simulation is calculated as

$$
\beta_{j}=\left(1-\frac{a+q}{j}\right) \beta_{j-1} .
$$

Let the general form of the $3 \mathrm{D}$ fractional order system be defined as

$$
\begin{aligned}
& D^{q_{x}}=A(x, y, z, w, t), \\
& D^{q_{y}}=B(x, y, z, w, t), \\
& D^{q_{z}}=C(x, y, z, w, t), \\
& D^{q_{w}}=D(x, y, z, w, t) .
\end{aligned}
$$


In order to simulate system (8) using GL method we use the discretization method discussed $[34,35]$,

$$
\begin{aligned}
x\left(t_{k}\right)= & A\left(x\left(t_{k-1}\right), y\left(t_{k-1}\right), z\left(t_{k-1}\right), w\left(t_{k-1}\right)\right) h^{q_{x}} \\
& -\sum_{j=1}^{N} \beta_{j}^{q_{x}} x\left(t_{k-j}\right), \\
y\left(t_{k}\right)= & B\left(x\left(t_{k-1}\right), y\left(t_{k-1}\right), z\left(t_{k-1}\right), w\left(t_{k-1}\right)\right) h^{q_{y}} \\
& -\sum_{j=1}^{N} \beta_{j}^{q_{y}} y\left(t_{k-j}\right), \\
z\left(t_{k}\right)= & C\left(x\left(t_{k-1}\right), y\left(t_{k-1}\right), z\left(t_{k-1}\right), w\left(t_{k-1}\right)\right) h^{q_{z}} \\
& -\sum_{j=1}^{N} \beta_{j}^{q_{z}} z\left(t_{k-j}\right), \\
w\left(t_{k}\right)= & C\left(x\left(t_{k-1}\right), y\left(t_{k-1}\right), z\left(t_{k-1}\right), w\left(t_{k-1}\right)\right) h^{q_{w}} \\
& -\sum_{j=1}^{N} \beta_{j}^{q_{w}} w\left(t_{k-j}\right),
\end{aligned}
$$

where $\beta$ is the binomial coefficients calculated using (11). The value of $N$ is taken as the truncation window size $L$ and as $k$ when all the available memory elements are used.

Let us define the FO2DOFSMA oscillator as

$$
\begin{aligned}
& D^{q_{x}} x=y \\
& D^{q_{y}} y=\left[\delta_{1} \sin \left(\omega_{1} t\right)-\left(\varepsilon_{1}+\varepsilon_{2} v_{21} \mu\right) y+\varepsilon_{2} v_{21} \mu z\right. \\
& \quad-\left[\left(\theta_{1}-1\right)+v_{21}{ }^{2} \mu\left(\theta_{2}-1\right)\right] x+v_{21}{ }^{2} \mu\left(\theta_{2}-1\right) z \\
& \quad+\phi_{1} x^{3}-\lambda_{1} x^{5}-\phi_{2} v_{21}{ }^{2} \mu(z-x)^{3} \\
& \left.\quad+\lambda_{2} v_{21}{ }^{2} \mu(z-x)^{5}\right], \\
& D^{q_{z}} z=w, \\
& D^{q_{w}} w=\left[\alpha_{21}{ }^{2} \delta_{2} \sin \left(\omega_{2} t\right)+\varepsilon_{2} v_{21} y\right. \\
& \quad-\left(\varepsilon_{2} \alpha_{21}+\varepsilon_{3} v_{21} \alpha_{32}\right) w^{2} v_{21}{ }^{2}\left(\theta_{2}-1\right) x \\
& \quad-\left[v_{21}{ }^{2}\left(\theta_{2}-1\right)+v_{21}{ }^{2} v_{32}{ }^{2}\left(\theta_{3}-1\right)\right] z \\
& +\phi_{2} v_{21}{ }^{2}(z-x)^{3}-\lambda_{2} v_{21}{ }^{2}(z-x)^{5}+\phi_{3} v_{21}{ }^{2} v_{32}{ }^{2} z^{3} \\
& \left.-\lambda_{3} v_{21}{ }^{2} v_{32}{ }^{2} z^{5}\right] .
\end{aligned}
$$

Using (9) in (10), the discrete form of the FO2DOFSMA is given by

$$
\begin{aligned}
& x\left(t_{k}\right)=\left(y\left(t_{k-1}\right)\right) h^{q_{x}}-\sum_{j=1}^{N} \beta_{j}^{q_{x}} x\left(t_{k-j}\right) \\
& y\left(t_{k}\right)=\left(\delta_{1} \sin \left(\omega_{1} t\right)-\left(\varepsilon_{1}+\varepsilon_{2} v_{21} \mu\right) y\left(t_{k-1}\right)\right. \\
& \quad+\varepsilon_{2} v_{21} \mu z\left(t_{k-1}\right)
\end{aligned}
$$

$$
\begin{aligned}
& -\left[\left(\theta_{1}-1\right)+v_{21}^{2} \mu\left(\theta_{2}-1\right)\right] x\left(t_{k-1}\right) \\
& +v_{21}^{2} \mu\left(\theta_{2}-1\right) z\left(t_{k-1}\right)+\phi_{1} x\left(t_{k-1}\right)^{3} \\
& -\lambda_{1} x\left(t_{k-1}\right)^{5}-\phi_{2} v_{21}{ }^{2} \mu\left(z\left(t_{k-1}\right)-x\left(t_{k-1}\right)\right)^{3} \\
& \left.+\lambda_{2} v_{21}^{2} \mu\left(z\left(t_{k-1}\right)-x\left(t_{k-1}\right)\right)^{5}\right) h^{q_{y}} \\
& -\sum_{j=1}^{N} \beta_{j}^{q_{y}} y\left(t_{k-j}\right) \\
& z\left(t_{k}\right)=\left(w\left(t_{k-1}\right)\right) h^{q_{z}}-\sum_{j=1}^{N} \beta_{j}^{q_{z}} z\left(t_{k-j}\right), \\
& w\left(t_{k}\right)=\left(\alpha_{21}^{2} \delta_{2} \sin \left(\omega_{2} t\right)+\varepsilon_{2} v_{21} y\left(t_{k-1}\right)\right. \\
& -\left(\varepsilon_{2} \alpha_{21}+\varepsilon_{3} v_{21} \alpha_{32}\right) w\left(t_{k-1}\right) \\
& +v_{21}^{2}\left(\theta_{2}-1\right) x\left(t_{k-1}\right) \\
& -\left[v_{21}{ }^{2}\left(\theta_{2}-1\right)+v_{21}{ }^{2} v_{32}{ }^{2}\left(\theta_{3}-1\right)\right] z\left(t_{k-1}\right) \\
& +\phi_{2} v_{21}^{2}\left(z\left(t_{k-1}\right)-x\left(t_{k-1}\right)\right)^{3} \\
& -\lambda_{2} v_{21}^{2}\left(z\left(t_{k-1}\right)-x\left(t_{k-1}\right)\right)^{5} \\
& \left.+\phi_{3} v_{21}{ }^{2} v_{32}{ }^{2} z\left(t_{k-1}\right)^{3}-\lambda_{3} v_{21}{ }^{2} v_{32}{ }^{2} z\left(t_{k-1}\right)^{5}\right) h^{q_{w}} \\
& -\sum_{j=1}^{N} \beta_{j}^{q_{w}} w\left(t_{k-j}\right)
\end{aligned}
$$

The value of $N$ is taken as the truncation window size $L$ and as $k$ when all the available memory elements are used.

For the parameters values given by (2) and Table 2, the FO2DOFSMA oscillator shows different behaviors as like the integer order SMA. For initial conditions $[0,0,0,1]$, commensurate order $q=0.99$, and step size $h=0.001$, the different behaviors of the FO2DOFSMA are given in Figure 4.

We study the bifurcation of the FO2DOFSMA with fractional order $q$ and parameter $\theta_{2}$. The bifurcation of the FO2DOFSMA oscillator with the fractional order $q$ is shown in Figure 5(a). The initial conditions are taken as $[0,0,0,1]$ and are reinitialized in end of each iteration to the final values of the state variables. The FO2DOFSMA shows chaotic oscillations for $q \geq 0.968$. Figure 5(b) shows the bifurcation of the oscillator with $\theta_{2}$ and shows chaotic oscillations for $0.47<\theta_{2} \leq 0.8,1.1<\theta_{2} \leq 1.6$. Hyperchaotic oscillations are seen for $0.8<\theta_{2} \leq 1.1$, quasiperiodic oscillations for $1.6<\theta_{2} \leq 2.2$, and period 4 oscillations for $2.2<\theta_{2} \leq 4$. For Figure 5(b), we used initial conditions without reinitialization. Figure 6 shows the bifurcation plots of the oscillator with reinitialization of the initial conditions. Figure 6(b) shows zoomed in portion of Figure 6(a) to shows the period doubling route to chaos. 
TABLE 2: Types of fractional order 2DOF SMA behavior.

\begin{tabular}{lcc}
\hline Parameters & Type of SMA behavior & Lyapunov exponents \\
\hline$\theta_{1}=1.5, \theta_{2}=3.5, \theta_{3}=1.5$ & Periodic & $-0.128 ;-0.140 ;-0.257 ;-0.275$ \\
$\theta_{1}=1.5, \theta_{2}=2, \theta_{3}=1.5$ & Quasiperiodic & $0 ;-0.209 ;-0.265 ;-1.272$ \\
$\theta_{1}=1.5, \theta_{2}=1.5, \theta_{3}=1.5$ & Chaotic & $0.378 ; 0 ;-0.438 ;-0.786$ \\
$\theta_{1}=1.5, \theta_{2}=1, \theta_{3}=1.5$ & Hyperchaotic & $0.453 ; 0.233 ;-0.651 ;-0.835$ \\
\hline
\end{tabular}
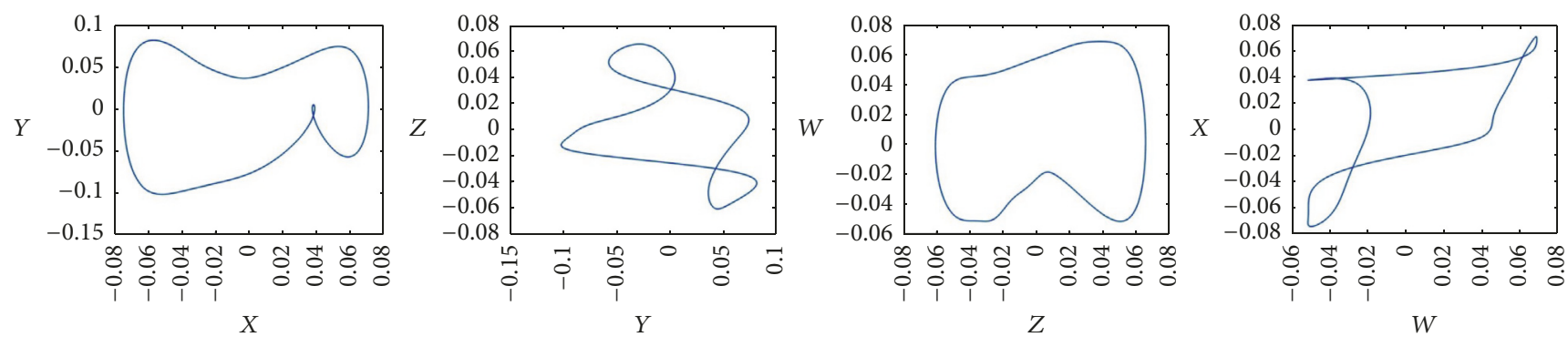

(a)
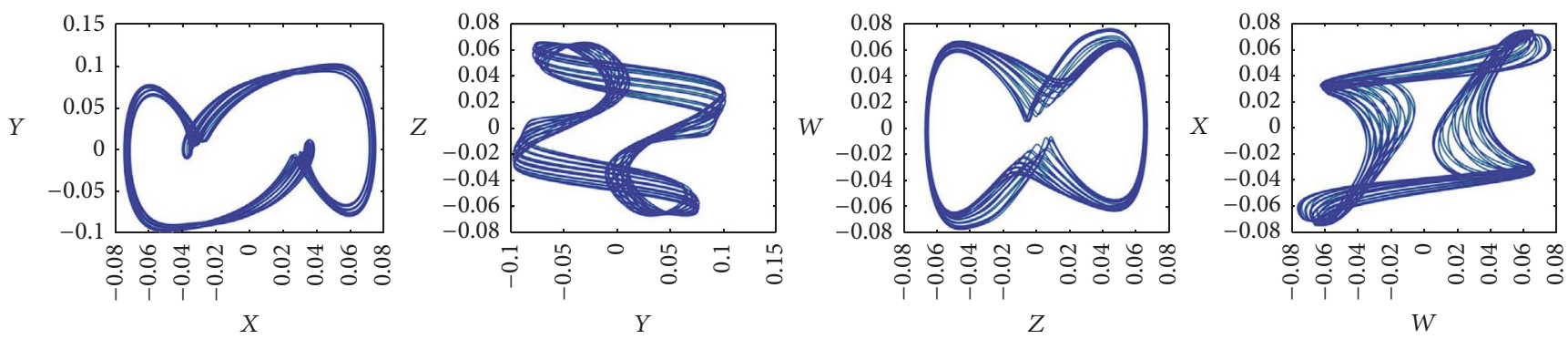

(b)
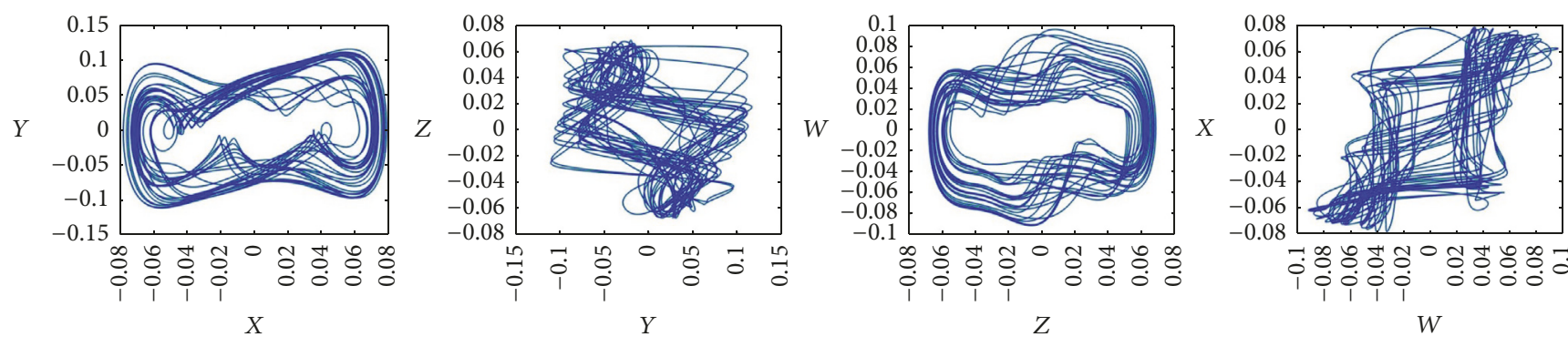

(c)
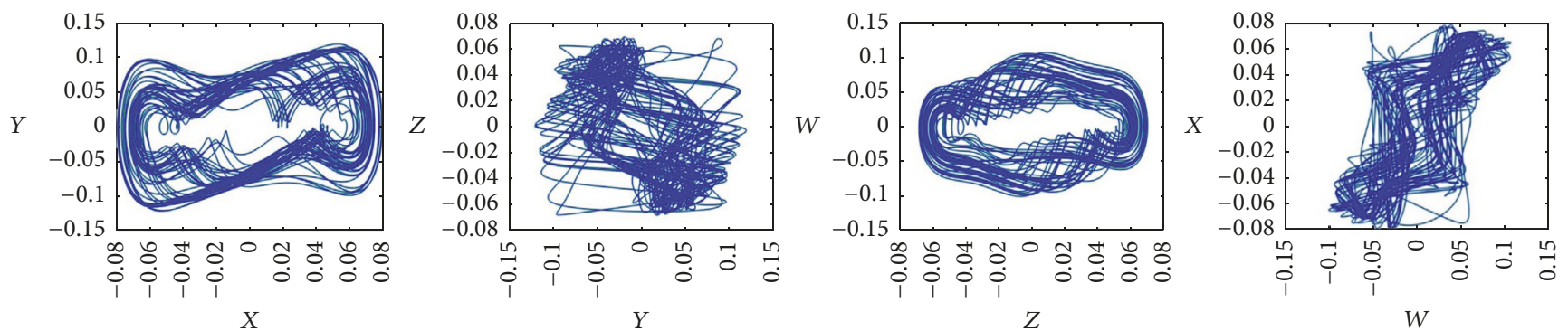

(d)

FIGURE 4: 2D phase portraits of the FO2DOFSMA oscillator: (a) periodic; (b) quasiperiodic; (c) chaotic; (d) hyperchaotic. 


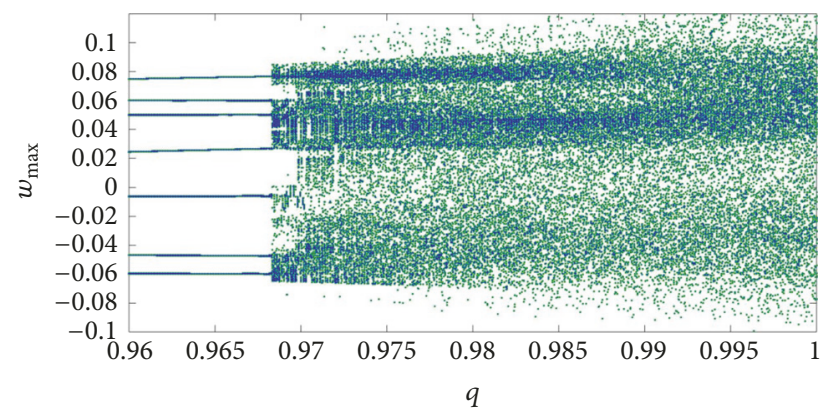

(a)

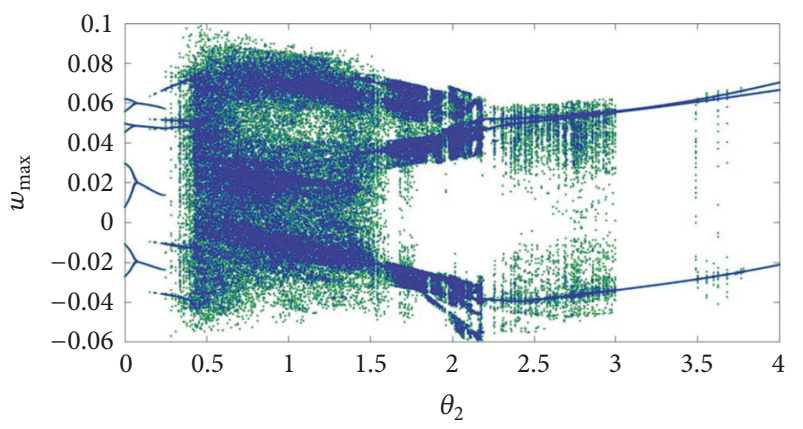

(b)

Figure 5: Bifurcation plots of 2DOFSMA oscillator with (a) commensurate fractional order $q$ and (b) parameter $\theta_{2}$ with $q=0.99$ and $\theta_{1}=\theta_{2}=1.5$. The initial conditions for plot (a) are taken as $[0,0,0,1]$ and are reinitialized in end of each iteration to the final values of the state variables and for plot $(b)$ the initial value is fixed at $[0,0,0,1]$.

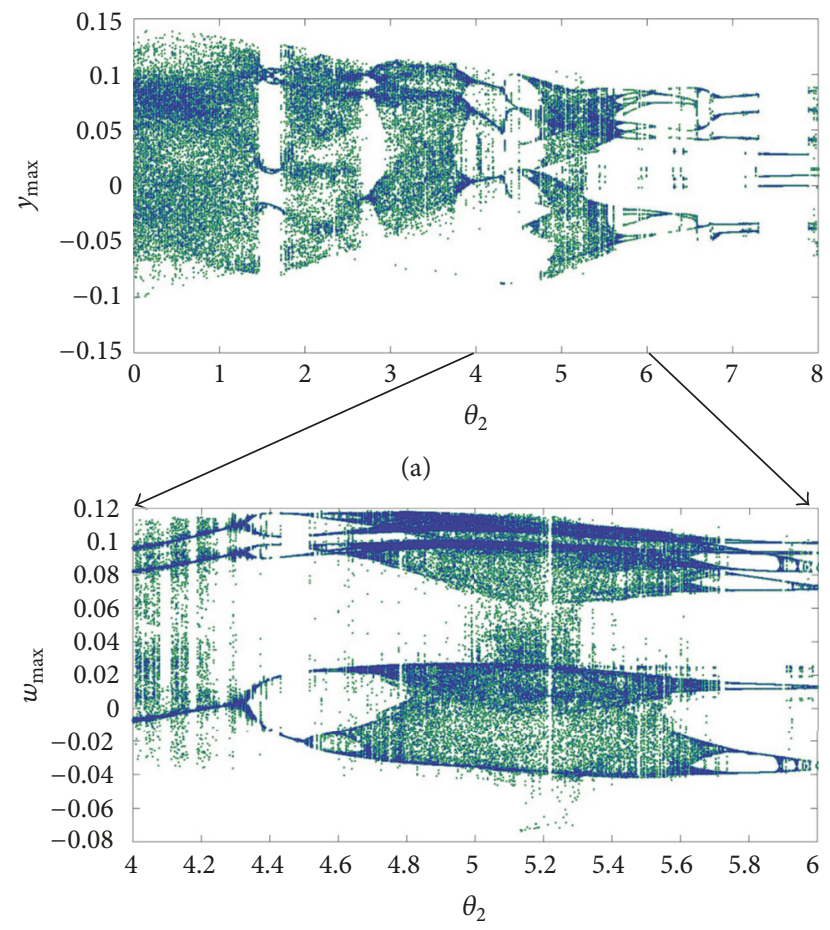

(b)

FIGURE 6: (a) Bifurcation of the FO2DOFSMA oscillator with parameter $\theta_{2}$ with $q=0.99$ and $\theta_{1}=\theta_{2}=1.5$. The initial conditions for are taken as $[0,0,0,1]$ and are reinitialized in end of each iteration to the final values of the state variables. (b) shows the zoomed in view of (a) between $4 \leq \theta_{2} \leq 6$.

\section{Conclusion}

A two-degree-of-freedom polynomial constitutive shape memory alloy oscillator is investigated and it is shown that periodic, quasiperiodic, chaotic, and hyperchaotic behaviors are shown by the oscillator for various values of the excitation parameters and temperatures. Bifurcation plots are derived to show the existence of chaotic and periodic oscillations. The Grünwald-Letnikov derivative is used to derive the fractional order model of the oscillator and bifurcation plots for fractional order and parameter are derived to study the dynamical behavior of the oscillator. Compared to the integer order model, the fractional order model shows more complex chaotic behavior and the quasiperiodic region is extended as chaotic regions and the oscillator shows more extended regions of chaos with parameters. This proves that the fractional order model shows more complex behavior and hence designing controllers to suppress chaos in fractional orders is quiet complicated.

\section{Conflicts of Interest}

The authors declare that there are no conflicts of interest in publishing of this paper.

\section{References}

[1] L. Wang and R. Melnik, "Dynamics of shape memory alloys patches with mechanically induced transformations," Discrete and Continuous Dynamical Systems - Series A, vol. 15, no. 4, pp. 1237-1252, 2006.

[2] M. A. Savi, M. A. N. Sa, A. Paiva, and P. M. C. L. Pacheco, "Nonlinear Dynamics and Chaos in a shape memory alloys oscillator," in Proceedings of the XII international symposium on Dynamic problems of Mechanics (DINAME), 2007.

[3] M. J. Mosley and C. Mavroidis, "Experimental nonlinear dynamics of a shape memory alloy wire bundle actuator," Journal of Dynamic Systems, Measurement, and Control, vol. 123, no. 1, pp. 103-112, 2001.

[4] L. Wang and R. V. N. Melnik, "Nonlinear dynamics of shape memory alloy oscillators in tuning structural vibration frequencies," Mechatronics, vol. 22, no. 8, pp. 1085-1096, 2012.

[5] M. A. Savi, "Nonlinear dynamics and chaos in shape memory alloy systems," International Journal of Non-Linear Mechanics, vol. 70, pp. 2-19, 2015.

[6] D. Bernardini and G. Rega, "Thermomechanical modelling, nonlinear dynamics and chaos in shape memory oscillators," Mathematical and Computer Modelling of Dynamical Systems, vol. 11, no. 3, pp. 291-314, 2005. 
[7] L. G. Machado, D. C. Lagoudas, and M. A. Savi, "Lyapunov exponents estimation for hysteretic systems," International Journal of Solids and Structures, vol. 46, no. 6, pp. 1269-1286, 2009.

[8] A. Paiva, M. A. Savi, A. M. B. Braga, and P. M. C. L. Pacheco, "A constitutive model for shape memory alloys considering tensile-compressive asymmetry and plasticity," International Journal of Solids and Structures, vol. 42, no. 11-12, pp. 3439-3457, 2005.

[9] M. A. Savi and Bragaw A. Mz, "Chaotic Vibration of an oscillator with shape memory systems," Journal of the Brazilian Society of Mechanical Sciences and Engineering, vol. 15, no. 1, p. 20, 1993.

[10] M. A. Savi and P. M. C. L. Pacheco, "Chaos and hyperchaos in shape memory systems," International Journal of Bifurcation and Chaos, vol. 12, no. 3, pp. 645-657, 2002.

[11] M. A. Savi, F. H. Pereira-Pinto, F. M. Viola et al., "Using 0-1 test to diagnose chaos on shape memory alloy dynamical systems," Chaos, Solitons \& Fractals, vol. 103, pp. 307-324, 2017.

[12] D. Bernardini and G. Rega, "Chaos robustness and strength in thermomechanical shape memory oscillators part II: Numerical and theoretical evaluation," International Journal of Bifurcation and Chaos, vol. 21, no. 10, pp. 2783-2800, 2011.

[13] A. Paiva and M. A. Savi, "An overview of constitutive models for shape memory alloys," Mathematical Problems in Engineering, vol. 2006, Article ID 56876, 30 pages, 2006.

[14] H. S. Oliveira, A. S. de Paula, and M. A. Savi, "Dynamical jumps in a shape memory alloy oscillator," Shock and Vibration, vol. 2014, Article ID 656212, 10 pages, 2014.

[15] Z. C. Feng and D. Z. Li, "Dynamics of a mechanical system with a shape memory alloy bar," Journal of Intelligent Material Systems and Structures, vol. 7, no. 4, pp. 399-410, 1996.

[16] M. W. Hirsch and S. Smale, Differential Equations, Dynamical Systems and Linear Algebra, Chapter 11, Academic Press, New York, NY, USA, 1974.

[17] T. T. Hartley, C. F. Lorenzo, and H. K. Qammer, "Chaos in a fractional order Chua's system," IEEE Transactions on Circuits and Systems I: Fundamental Theory and Applications, vol. 42, no. 8, pp. 485-490, 1995.

[18] T. Manzoor, Z. Mehmood, M. A. Zahid, S. T. Mohyud-Din, H. Manzoor, and S. Hashmi, "A note on fractional order in thermoelasticity of shape memory alloys' dampers," International Journal of Heat and Mass Transfer, vol. 114, pp. 597-606, 2017.

[19] M. A. Ezzat and A. S. El Karamany, "Theory of fractional order in electro-thermoelasticity," European Journal of Mechanics A/Solids, vol. 30, no. 4, pp. 491-500, 2011.

[20] Y. J. Yu, X. G. Tian, and T. J. Lu, "Fractional order generalized electro-magneto-thermo-elasticity," European Journal of Mechanics - A/Solids, vol. 42, pp. 188-202, 2013.

[21] L. G. Machado, M. A. Savi, and P. M. C. L. Pacheco, "Nonlinear dynamics and chaos in coupled shape memory oscillators," International Journal of Solids and Structures, vol. 40, no. 19, pp. 5139-5156, 2003.

[22] A. Wolf, J. B. Swift, H. L. Swinney, and J. A. Vastano, "Determining Lyapunov exponents from a time series," Physica D: Nonlinear Phenomena, vol. 16, no. 3, pp. 285-317, 1985.

[23] K. Diethelm, The analysis of fractional differential equations: An application-oriented exposition using differential operators of Caputo type, Springer, 2010.

[24] D. Xue, C. Zhao, and Y. Chen, "A modified approximation method of fractional order system," in Proceedings of the 2006 IEEE International Conference on Mechatronics and Automation, ICMA 2006, pp. 1043-1048, China, June 2006.
[25] D. Baleanu and S. I. Muslih, "Nonconservative systems within fractional generalized derivatives," Journal of Vibration and Control, vol. 14, no. 9-10, pp. 1301-1311, 2008.

[26] C.-m. Chen, F. Liu, and K. Burrage, "Finite difference methods and a Fourier analysis for the fractional reaction-subdiffusion equation," Applied Mathematics and Computation, vol. 198, no. 2, pp. 754-769, 2008.

[27] T. A. Langlands and B. I. Henry, "The accuracy and stability of an implicit solution method for the fractional diffusion equation," Journal of Computational Physics, vol. 205, no. 2, pp. 719-736, 2005.

[28] F. Mainardi, A. Mura, G. Pagnini, and R. Gorenflo, "Subdiffusion equations of fractional order and their fundamental solutions," Mathematical Methods in Engineering, pp. 23-55, 2006.

[29] Y. Zhou, J. Wang, and L. Zhang, Basic Theory of Fractional Differential Equations, World Scientific. ISBN: 978-981-3148-185 (ebook), 2016.

[30] D. Baleanu, K. Diethelm, E. Scalas, and J. J. Trujillo, "Fractional calculus: models and numerical methods," in Series on Complexity, Nonlinearity and Chaos, vol. 5, World Scientific. ISBN: 978981-4458-63-4 (ebook), 2017.

[31] C. L. MacDonald, N. Bhattacharya, B. P. Sprouse, and G. A. Silva, "Effcient computation of the Grünwald-Letnikov fractional diffusion derivative using adaptive time step memory," Journal of Computational Physics, vol. 297, pp. 221-236, 2015.

[32] S. B. Yuste and L. Acedo, "On an explicit finite difference method for fractional diffusion equations," SIAM J. Numer. Anal, vol. 42, no. 5, pp. 1862-1874, 2005.

[33] I. Podlubny, Fractional Differential Equations, Academic Press, San Diego, Calif, USA, 1999.

[34] M. F. Tolba, A. M. AbdelAty, N. S. Soliman et al., "FPGA implementation of two fractional order chaotic systems," $A E \ddot{U}$ - International Journal of Electronics and Communications, vol. 78, pp. 162-172, 2017.

[35] I. Petráš, Fractional-Order Nonlinear Systems. Nonlinear Physical Science, Springer ISBN 978-3-642-18100-9, Berlin, Heidelberg, 2011. 


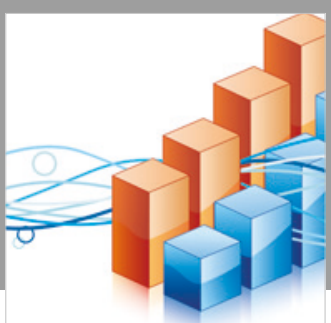

Advances in

Operations Research

\section{-n-m}
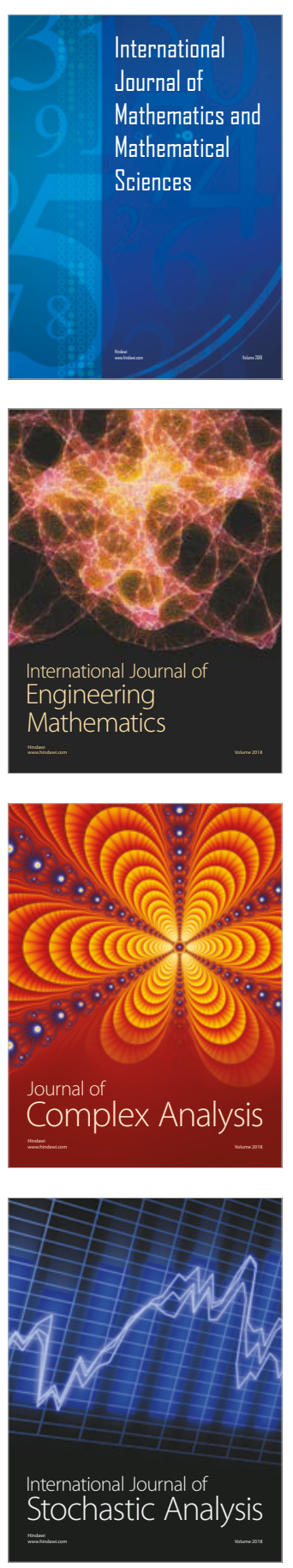
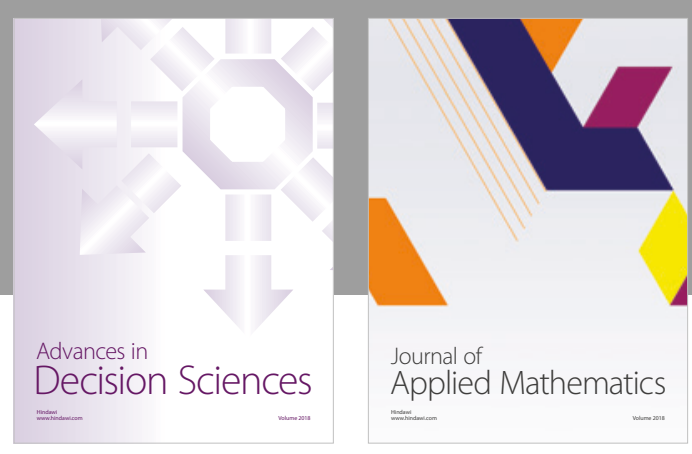

Journal of

Applied Mathematics
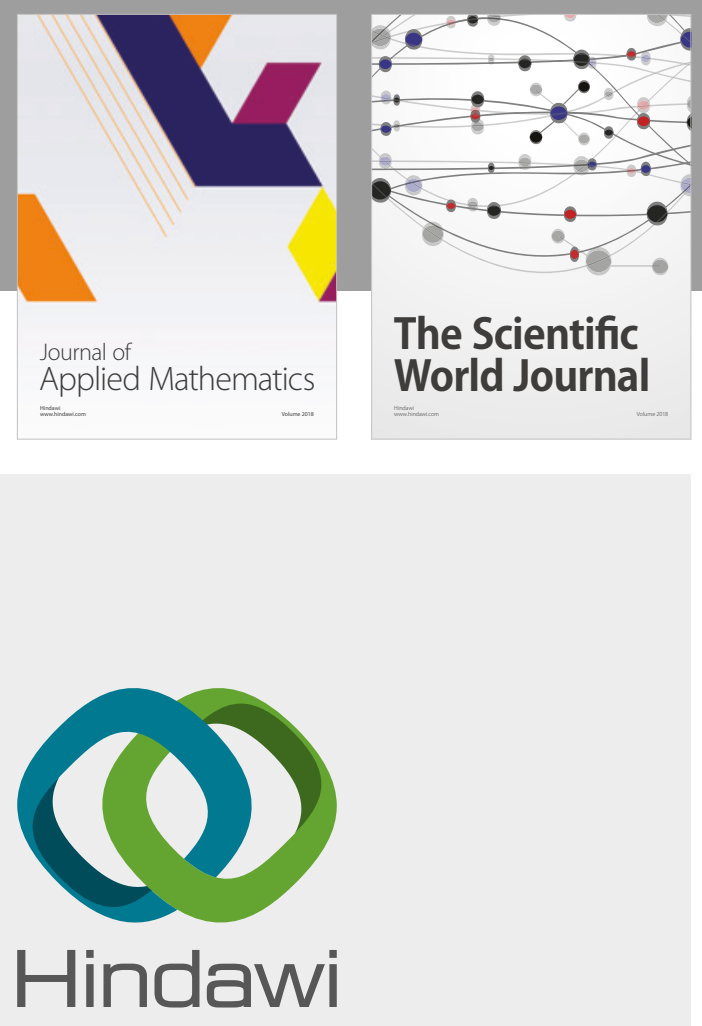

Submit your manuscripts at

www.hindawi.com

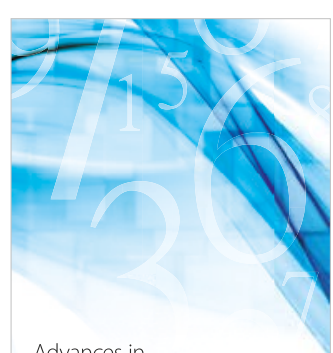

Advances in
Numerical Analysis
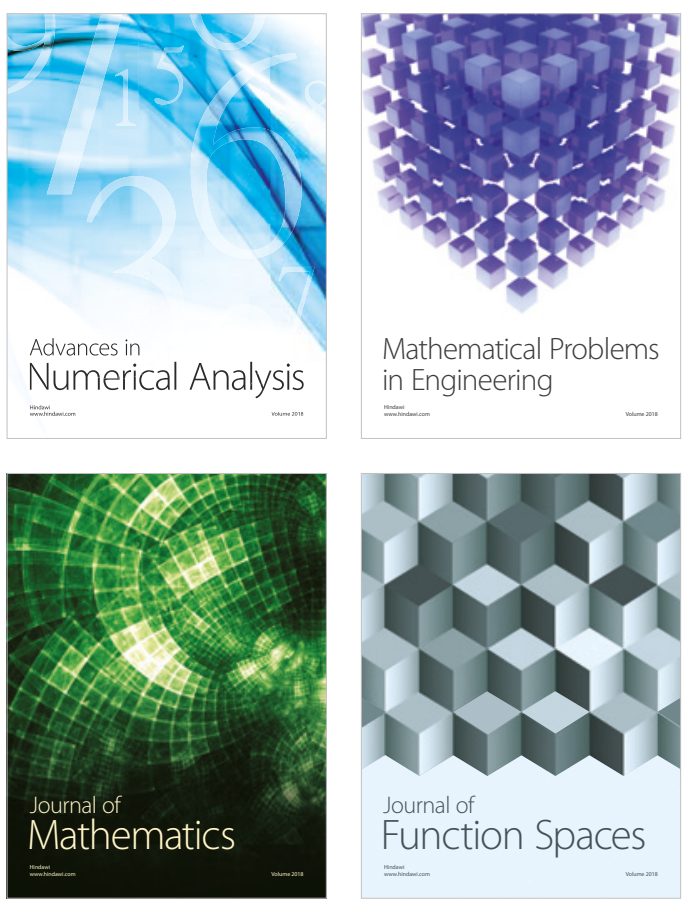

Mathematical Problems in Engineering

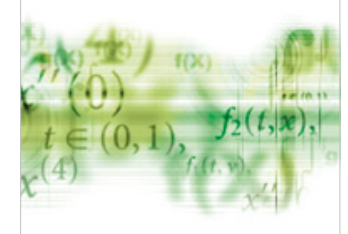

International Journal of

Differential Equations

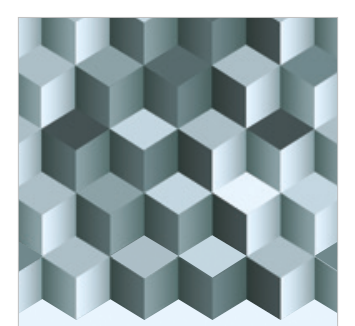

Journal of

Function Spaces
The Scientific

World Journal

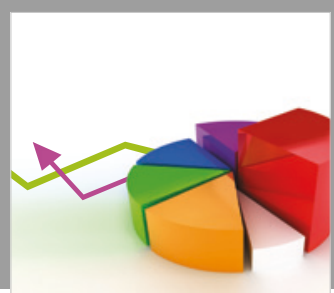

Journal of

Probability and Statistics
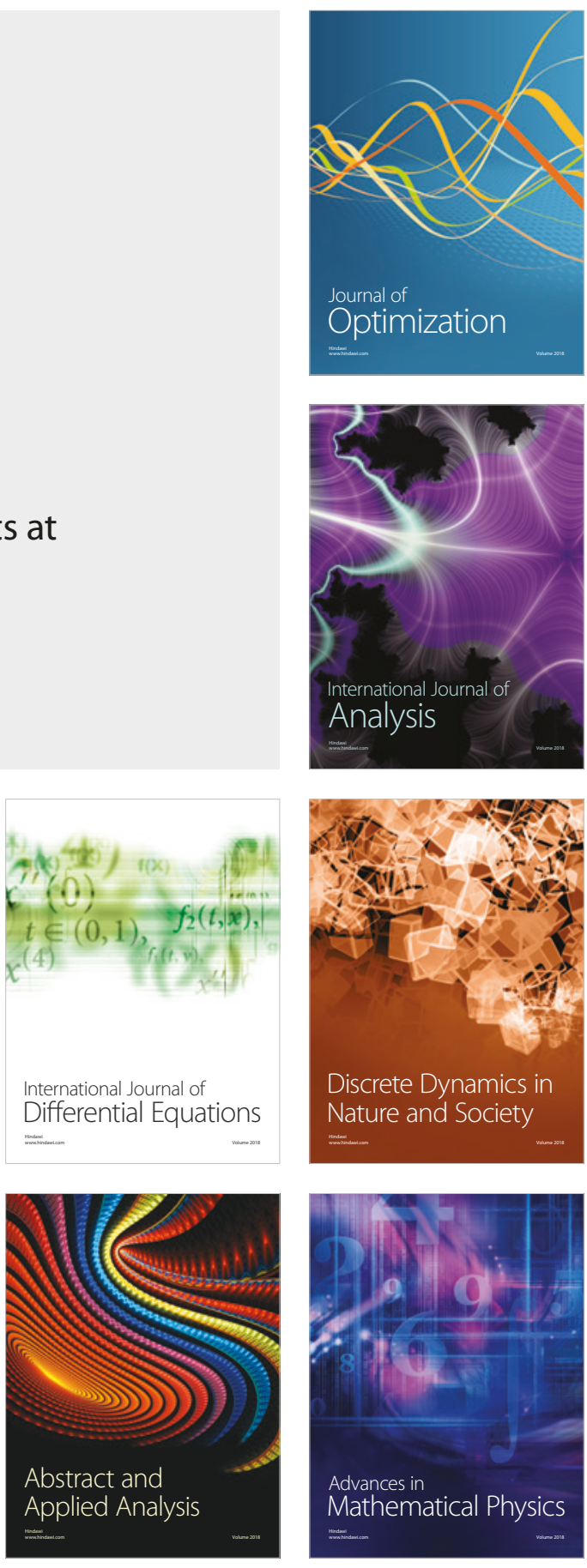